Itinéraires Itinéraires

Littérature, textes, cultures

2010-2 | 2010

Les blogs

\title{
Penser la forme des blogs, entre générique et génétique
}

Étienne Candel

\section{(2) OpenEdition}

Journals

Édition électronique

URL : http://journals.openedition.org/itineraires/1932

DOI : 10.4000/itineraires.1932

ISSN : 2427-920X

Éditeur

Pléiade

\section{Édition imprimée}

Date de publication : 1 juillet 2010

Pagination : 23-31

ISBN : 978-2-296-12012-9

ISSN : 2100-1340

\section{Référence électronique}

Étienne Candel, «Penser la forme des blogs, entre générique et génétique », Itinéraires [En ligne], 2010-2 | 2010, mis en ligne le 01 juillet 2010, consulté le 30 avril 2019. URL : http://

journals.openedition.org/itineraires/1932 ; DOI : 10.4000/itineraires.1932

\section{(ब) $(\Theta \Theta$}

Itinéraires est mis à disposition selon les termes de la licence Creative Commons Attribution - Pas d'Utilisation Commerciale - Pas de Modification 4.0 International. 


\title{
Penser la forme des blogs, entre générique et génétique
}

\begin{abstract}
The aim of this paper is to allow a more precise understanding of the social conception of blogs. The common definition of blogs as online personal diaries is limited to covering the multifarious ways they are used. A blog is a formal object that is characterized by its ability to adapt itself to different projects : as the social conception of blogs is related to writing as an act of appropriation, so the paper focuses on the benefits of a genetic perspective and studies what blogs refers to in the field of communication and social actions.
\end{abstract}

Keywords : editing semiotics, blogs, genre, uses and gratifications, writing Mots clés : sémiotique éditoriale, blogs, genre, usages, écriture

Qu'il y ait un phénomène social et médiatique des blogs ne fait aucun doute; mais si l'on se demande ce que recouvre ce phénomène, on est gêné parce qu'à cette pratique, il est difficile d'attribuer des caractéristiques génériques. On remarque, d'une part, une tendance à définir le blog comme un « journal personnel en ligne », et d'autre part, on peut être frappé par le nombre important de blogs qui ne relèvent pas de cette qualification générique. On a affaire à des actions de publication et de médiatisation, c'est une certitude; mais la question me semble se poser de savoir si ces pratiques sont appréhendables comme une pratique générique, c'est-à-dire de savoir si elles sont subsumables sous une seule catégorie textuelle.

Questionner ce rapport entre une pratique sociale et sa compréhension comme genre, c'est en fait chercher à comprendre si l'on a réellement affaire à une catégorie du scriptural, quand on emploie le terme de blog, ou si ce terme n'est qu'une étiquette placée sur une multiplicité de cas divers, hétérogènes et irréductibles. Dans une certaine mesure, on pourrait dire que la question de la généricité (ou non) du blog est celle de sa définition même : 
en se demandant « qu'est-ce qu'un blog? », on cherche à circonscrire un phénomène attesté en interrogeant son essence communicationnelle.

À cette première tension (qu'on pourrait dire essentialiste, ou ontologique, puisqu'il s'agit de savoir quel est l'être des blogs) qui distingue l'approche du blog comme genre de son analyse comme pratique, il faut ajouter un second questionnement problématique : l'existence phénoménale des blogs en tant qu'ils sont des textes est manifeste; mais elle est doublée d'un second mode d'existence, qui est son existence discursive et idéologique comme représentation sociale. On ne peut faire l'économie, dans le cas des blogs, de l'intense production de discours et de représentations qui les constitue en « phénomène social » ou en « phénomène médiatique ». Ainsi, si l'on cherche à définir le blog comme genre, on doit prendre en compte non seulement les pratiques génériques à proprement parler, mais aussi les discours et les pratiques de ce qui constitue son escorte, son accompagnement, et qui influe sur le travail de l'usage par le genre.

Je proposerai donc d'observer les blogs d'abord comme ce qu'ils sont le plus manifestement : des sites Internet, rendus caractéristiques par des formes éditoriales ${ }^{1}$ et par des infrastructures techniques spécifiques. L'examen de ces formes me mènera à observer que le blog est une forme qui se prête à une grande variété d'usages, rendant très difficile l'approche générique.

Dans un deuxième temps, j'aborderai la question du contexte social du développement de cette forme éditoriale, et des implications de ce contexte dans les pratiques du texte. L'examen des idéologies liées à la qualification générique des blogs m'amènera à proposer la perspective d'une approche génétique centrée sur l'activité d'écriture. Cette perspective me semble de nature à expliquer la récurrence de la définition du blog comme « journal personnel en ligne ».

\section{L'insaisissable genre d'une forme éditoriale}

\section{Le blog est une forme éditoriale...}

\section{Un modèle canonique}

Un blog peut se définir d'abord comme un site présentant une forme éditoriale spécifique. Décrire le blog de la sorte, c'est reconnaître, derrière la

1. Une forme éditoriale est une organisation visuelle de la page qui permet d'en structurer et d'en organiser le sens. L'analyse des formes éditoriales permet d'appréhender les textes non seulement par leurs « contenus », leurs dimensions linguistique et discursive, mais aussi par le biais de leurs supports, de leur organisation graphique - elle permet donc de prendre en compte leur complexité, leur hétérogénéité sémiotiques et la dimension fondamentalement sociale des textes comme objets de la culture. 
diversité des emplois et des cas, une structure canonique unifiée, isolable. La centralité sémiotique de l'énonciateur principal (l'auteur-éditeur du blog), la liste de liens, le calendrier permettant un accès aux archives, l'organisation verticale antichronologique des textes rédigés, l'inscription de la polyphonie dans la page par le biais des zones de commentaire, tout cela compose les attributs canoniques de la forme. Un blog ressemblera ensuite de manière plus ou moins complète à ce modèle canonique - un élément peut manquer, des aménagements peuvent survenir - mais on a bien, avec cette approche, une définition du blog à travers ses propriétés formelles.

\section{Iconicité de la forme éditoriale}

Un autre type d'élément devrait ici intervenir, c'est le fait qu'un blog se reconnaît aussi à sa conception graphique générale, à son design. C'est ce qui contribue à donner l'impression non seulement d'une forme, mais aussi d'une certaine uniformité des blogs. À ce titre, il est particulièrement frappant de procéder à ce qu'on pourrait appeler un test de reconnaissance : un blog dont tous les textes seraient brouillés, remplacés par exemple par ce que dans l'édition on appelle du faux-texte, ou par une langue étrangère, ou même par des blocs grisés sans caractères distincts, apparaîtra quand même, de loin, comme un blog ${ }^{2}$. C'est que le blog se reconnaît spontanément en raison de l'image du texte qu'il produit ${ }^{3}$. On observe ainsi, d'une part, l'existence d'une structure sous-jacente qui organise l'écriture comme un ensemble de fonctionnements éditoriaux et, d'autre part, une récurrence des modes de présentation et d'affichage. L'un et l'autre de ces aspects sont en fait solidaires, car ils relèvent de l'impact sémiotique, de l'impact visible, lisible et interprétable, de ce que Yves Jeanneret et Emmanuël Souchier appellent les architextes ${ }^{4}$ : les plates-formes de blogs proposent des modèles, des gabarits, des patrons typiques, qui donnent une certaine systématicité, une certaine régularité à toutes les configurations formelles de blog.

L'impact des architextes est lié avant tout au fait que l'écriture logicielle a pour fonction, dans les blogs, de simplifier et de systématiser les tâches

2. L'idée de ce test a été proposée par Marie Després-Lonnet dans le cadre du programme blanc TRAMEDWEB de l'ANR, qui rassemble des chercheurs du GRIPIC (CELSA - Université de Paris-Sorbonne), du MoDyCo (Université Paris X), du GERIICO (Université Lille 3) et du Laboratoire Culture et Communication de l'Université d'Avignon.

3. Cette notion d'image du texte a été proposée par Emmanuël Souchier, «L'image du texte. Pour une théorie de l'énonciation éditoriale », Cahiers de médiologie, n 6, décembre 1998. 4. Les logiciels d'écriture dans les médias informatisés sont des textes qui permettent la production d'autres textes, des textes qui sont à leur origine et qui tendent à en commander les réalisations (Voir Yves Jeanneret et Emmanuël Souchier, « Pour une poétique de l'écrit d'écran », Xoana. Images et sciences sociales, n 6-7, 1999, p. 97-107). C'est typiquement le cas des traitements de texte par exemple, mais aussi des outils permettant la production des blogs. Un architexte permet, engage et conditionne la production d'autres textes, tout en étant lui-même un écrit chargé de sens. 
d'édition et de mise en forme. Les « CMS », ou systèmes de management de contenus, qui automatisent la gestion des bases de données et les processus de mise en page des contenus, déchargent notamment l'utilisateur de la nécessité d'une connaissance du code HTML. Et en simplifiant ces tâches, ils contraignent et limitent les possibilités effectives de mise en forme et d'organisation éditoriale tout en proposant une accessibilité accrue aux modes de publication en ligne ${ }^{5}$. Ainsi, un impératif de simplicité et de manipulabilité se trouve directement en jeu dans la reconnaissance du blog comme forme. Dans ce qu'on appelle les « plates-formes de blogs », on peut constater la récurrence du recours à des modèles préconstruits pour la présentation, la mise en page, le design graphique de ces sites. Et au niveau de la forme se jouent à la fois la question de la manipulation des techniques et celle de la standardisation et de l'industrialisation des médiations culturelles.

Les blogs sont donc :

(i) des sites Internet spécifiques, se singularisant par leur forme éditoriale relativement stabilisée, récurrente et fortement reconnaissable;

(ii) des formes éditoriales dont la manipulation est simplifiée car elle repose sur des modes d'édition et de publication standardisés.

On perçoit que l'idée de publier de manière simplifiée est l'un des éléments récurrents du discours social sur les blogs, et qu'elle inspire à la fois les modes de manipulation et la structuration technique de ces objets. On peut en présumer l'importance idéologique et doxique de la promesse d'autopublication dont sont porteurs les blogs. C'est sans doute aussi la représentation sociale qui a le plus contribué à faire le succès de cette forme éditoriale : les « formes » seraient données, et l'internaute, semble-t-il, n'aurait qu'à fournir les « contenus ». Cette séparation actée entre forme et fond tend à manifester combien, dans leur essence de forme éditoriale, les blogs ne peuvent être considérés comme un genre : les thématisations, la tonalité, les grands déterminants de l'écriture restent par principe à la discrétion des utilisateurs.

\section{...marquée par une multiplicité d'usages différents}

\section{Une forme qui ne se mêle guère de contenus}

Si ces éléments ne suffisent pas à faire du blog un genre à part entière, c'est parce que ce type particulier de forme agit comme une proposition de publication, comme un mode de manipulation de la fonction éditoriale, qui ne se mêle guère des contenus; on peut même dire que le contenu est

5. C'est notamment l'apport de Valérie Jeanne-Perrier d'avoir montré cette ambiguïté structurante des CMS, qui les inscrit entre facilité et contrainte. Valérie Jeanne-Perrier, « L'écrit sous contrainte : les systèmes de management de contenu (CMS) », Communication \& Langages, $\mathrm{n}^{\circ} 146,2005$, p. 71-81. Valérie Jeanne-Perrier, « Des outils d'écriture aux pouvoirs exorbitants? », Réseaux, n 137, dossier « Autopublications », 2006, p. 97-131. 
censé relever de la liberté, de la créativité et de la volonté de l'utilisateur, la forme du blog se présentant comme un appel à l'inspiration et à la créativité individuelles. Cet appel à l'utilisateur inspiré dépend aussi d'un marché : les plates-formes sont, en général, des « solutions » distribuées par des entreprises qui ont tout à gagner à exalter la créativité et la liberté des utilisateurs ${ }^{6}$. En opérant une scission entre les formes - qui sont prises en charge par la proposition technique d'automatisation et de systématisation - et les contenus - laissés à la discrétion éclairée de l'internaute -, la publication sur Internet apparaît sous l'aspect d'une négation paradoxale de la fonction éditoriale : en effet, le texte vient se glisser, vient couler ${ }^{7}$ dans les formes préconstruites, comme si la médiation assurée par ces dernières était négligeable, comme si elles étaient neutres, comme si elles n'influençaient pas la réalisation sémiotique du texte. Les utilisateurs produisent du contenu, qu'ils « versent » dans les formes proposées, supposées neutres.

\section{Des contenus multiples}

Sous l'effet de cet imaginaire éditorial, des blogs se trouvent consacrés à tous types de sujets, mais aussi à tous types de matériaux langagiers.

Les blogs peuvent ainsi être le fait de différents types d'énonciateurs. Le blog « perso » relève normalement de l'intervention d'un privatus, d'un « simple particulier ». Mais l'auteur peut être une « personne morale » (une entreprise, voire une marque) se donnant une forme d'incarnation. Il faut d'ailleurs souligner que les mises en scène et les positionnements discursifs peuvent être nombreux : les blogs d'artistes, de professeurs, par exemple, sont faits par leurs auteurs en cette qualité, et ce n'est là qu'un des rôles sociaux de la personne qui assume cette auctorialité.

Le blog par ailleurs tend à déployer différents types de matériaux langagiers : du verbal, du pictural, du sonore (« radioblog »), de l'image animée (« vidéoblog »)... et différents types de genres textuels, avec des hybridations possibles (dans le cas des blogs-CV par exemple).

On pourra ajouter à cela que les blogs peuvent aussi travailler différents types de thématisations et d'objets référents ${ }^{8}$ : un champ de pratiques (les recettes de cuisine par exemple dans http://scally.typepad.com/cest_moi qui_lai_fait/), un domaine d'activité (comme le marketing dans http://blogweb-marketing.fr/), la vente d'un livre ou la promotion d'un produit culturel (blog de Thomas Clément, auteur de Les Enfants du plastique : http://clement.blogs.com/), une vie au quotidien (comme dans les nombreux blogs

6. On se souvient par exemple du «Jusqu'où irez-vous? » qui fut un temps le slogan de Microsoft.

7. Cette image d'un texte-fluide a été elle aussi proposée par Marie Després-Lonnet au cours des échanges sur le programme ANR TRAMEDWEB mentionné ci-dessus.

8. La véritable question serait surtout de savoir s'il existe des domaines insusceptibles d'une thématisation par le blog. 
de femmes enceintes : http://mini-maman.skyrock.com/), une ville (www. monputeaux.com, le blog fameux de Christophe Grébert), la politique, des blagues (http://humour-blague.com/blog/), des fictions ou récits (http://maguerre.over-blog.com/).

\section{Uniformité et polyvalence}

Si l'on prend ce dernier exemple du blog de récit, il y a quelque chose de paradoxal dans le fait que l'ordre antichronologique du blog vient contredire de manière radicale l'ordre chronologique du récit. L'existence attestée de tels blogs alors que le format type du blog paraît manifestement inadapté (mais adaptable, réversible, cependant), révèle une surdétermination de la pratique par l'idéologie. L'idée de la simplicité et de la manipulabilité des blogs tend à les définir comme une forme sans genre, une forme libre des déterminations proprement textuelles, une forme dont le propre serait précisément de pouvoir tout faire. La forme est vicariante, c'est-à-dire qu'elle apparaît comme radicalement polyvalente, mobilisable pour tout type d'énonciateur, et donc pour tout type de projet textuel. On peut encore reformuler cette proposition dans un autre sens : la forme éditoriale du blog serait une forme qui dépasserait les pratiques génériques pour la raison qu'elle prétendrait pouvoir les accomplir toutes.

De la sorte, si le blog est homogène par sa forme, il est hétérogène par ses relations génériques, et cette hétérogénéité apparaît essentielle, définitoire. La vicariance de la forme, son caractère de forme-à-tout-faire inscrit l'hétérogénéité et la polyvalence dans la conception même de l'objet. Si le blog apparaît formellement comme relevant d'un pouvoir-faire (le pouvoir de publier et d'éditer soi-même ses textes), c'est parce qu'il ne serait pas contraint par un devoir-être. La pratique d'édition et de publication vient donc prendre en quelque sorte le relais de la pratique générique.

Tout cela mène à affirmer qu'il n'y a pas de genre du blog à proprement parler, mais une forme blog fragmentée en différents usages, qui, eux, participent de certains genres (comme le journal, l'album, etc.). Il n'y aurait pas de genre du blog, mais ce que l'on pourrait appeler une génétique, c'est-à-dire une activité spécifique et assez intense d'appropriation de la forme par les usages, qui la plient et la soumettent à l'inscription générique de textes.

\section{Le blog, un référent social}

\section{Comment les pratiques génériques viennent au blog}

\section{La dynamique de l'usage}

Cette idée d'une vicariance de la forme éditoriale sur Internet, on la retrouve aussi, dans une certaine mesure, dans le cas des forums, par exemple : 
apparemment, ces formes éditoriales ont la propriété de pouvoir se prêter à tous types de mise en pratique, comme si leur propre était dans la dynamique de l'usage, qui les élabore en formes adaptables, maniables, détournables. Dans leur conception même, dans leur nature, dans leur essence, ces formes semblent avoir pour spécificité d'être accommodables. Leur propre serait ainsi précisément de ne pas avoir de propre. Un blog d'enseignant pourra être équipé d'une manière particulière, par exemple pour empêcher l'envoi de messages ou de commentaires - cet « équipement » étant alors de l'ordre de l'usage plus que du dispositif. Les appropriations possibles supposent donc l'assomption ou la désactivation, dans des gammes de pratiques proposées, de certaines fonctionnalités. Les pratiques inscrites dans les plates-formes de blogs composent des sortes d'injonctions et de contraintes, en ce que ces formes conditionnent l'action, mais elles ne le sont que de manière limitée, parce qu'elles sont modifiables. La manipulabilité et la vicariance reposent sur une relative souplesse du cadre éditorial.

\section{Le blog, objet de discours et d'idéologies}

Mais parallèlement à ces éléments, on doit remarquer que les blogs sont entourés de discours et de pratiques, c'est-à-dire que ce phénomène social est aussi un phénomène discursif, médiatique et idéologique. La forme éditoriale est prise dans un contexte idéologique et dans un contexte d'appropriation extrêmement denses. Des discours et des représentations disent le blog, en déterminent l'usage à partir d'une pensée de ce que cette forme est censée proposer, et de ce à quoi elle est censée se prêter particulièrement.

Cet usage est décrit avec une certaine vigueur dans la récurrence d'une définition du reste très imparfaite, très insatisfaisante du point de vue théorique : le blog serait un « journal personnel en ligne ». Ce qu'une telle qualification montre, c'est qu'il y aurait une tendance, une propension à faire du blog la forme propre d'une écriture du sujet, d'une écriture de l'intime ou de la personne, publiée sur Internet. Certes, on a vu que cet usage est loin d'être exclusif, et qu'une telle définition est inadéquate; mais ce qui en ressort, c'est aussi que le blog apparaît, à terme, comme un objet que l'on paraît vouloir ancrer d'abord dans une pratique de l'écriture personnelle : le blog serait en somme délié de sa définition comme forme éditoriale, au profit d'une définition comme pratique d'écriture. Il s'agit là d'une prédilection dans la destination supposée du blog, une prédilection qui est le composite d'une série de pratiques effectives, d'un ensemble de représentations, et d'une manière de penser les fonctions éditoriales de la forme. 


\section{Le blog comme référent idéologique et comme usage}

\section{La portée instituante des représentations sociales}

Le discours social sur les blogs est donc à prendre en compte, non uniquement en ce qu'il alimente quantitativement le phénomène, mais aussi en ce qu'il détermine la manière dont l'activité d'écriture et de publication peut être acquise et assumée. Les discours sur les blogs, au nombre desquels on doit compter les définitions inadéquates qui fleurissent dans la presse ou dans tout autre type de discours, sont à prendre en considération : ces représentations circulantes, triviales, ces définitions nombreuses et hétérogènes composent un imaginaire puissant de la forme et des usages auxquels elle serait associée de manière privilégiée.

\section{Génétique et éthique de l'usage}

Alors que la pratique du blog n'est pas une pratique directement générique, la définition du blog comme journal personnel en ligne me parait ainsi impliquer un ensemble de pratiques génétiques, dans lesquelles les différents genres mobilisés et accommodés dans l'usage imposent un ethos et une éthique de l'écriture. Cette éthique des blogs, on en trouve des traces par exemple dans un cas d'école du marketing numérique, celui de la marque Vichy qui avait créé un " faux blog » qui mettait en scène une prétendue consommatrice racontant pas à pas sa découverte des produits de la marque. Le faux-semblant avait fait scandale auprès de certains blogueurs ${ }^{9}$.

On voit que, dans ce cas, la forme blog se voit attribuer un impératif de véracité, d'authenticité, de transparence. La pratique de la forme est liée ici de manière rigoureuse à une éthique de l'écriture. Une représentation idéologique et morale du blog en fait le lieu de la véracité : comment comprendre cette éthique de l'écriture? Ce qui se passe, c'est qu'une normativité de la pratique se dégage à partir d'une référence à une conception générique : les usagers sont incités à adopter, dans leurs pratiques du blog, un comportement et une morale issus d'une tradition scripturale. Et en effet, les genres de l'écriture du moi - autobiographie, journal intime - sont des genres surdéterminés par la notion de véracité ${ }^{10}$. En empruntant à la pratique générique, en s'assimilant par prédilection au genre autobiographique, le blog engage ainsi une pratique génétique, c'est-à-dire une morale de l'écriture. Il n'y a donc pas de genre

9. Pour plus d'informations sur ce « cas d'école » du « marketing numérique », voir par exemple Verbal Kint, Blog corporate 2.0 : retour sur le "Journal de ma peau", blog des laboratoires Vichy », VerbalKint, 17 décembre 2007, http://www.verbalkint.net/BLOG-CORPORATE2-0-RETOUR-SUR-LE-JOURNAL-DE-MA-PEAU-,-BLOG-DES-LABORATOIRESVICHY_a471.html, consulté le 7 avril 2010.

10. C'est bien là en effet l'objet de ce que Philippe Lejeune nomme le « pacte autobiographique ». Philippe Lejeune, Le Pacte autobiographique, Paris, Seuil, 1975. 
du blog, mais il y a une sédimentation de pratiques génériques convoquées dans l'écriture, donc dans la genèse des textes. Dans la sémiosis sociale, une valeur et une prédilection sont attribuées aux blogs, qui sont pensés selon le modèle convoqué du journal ou de l'autobiographie.

Cet exemple de la référence au genre autobiographique est particulièrement important, parce qu'il montre que le blog se définit comme une forme éditoriale qui est sans inscription générique, mais dont la polyvalence engage en fait des pratiques issues des genres. Les genres sont convoqués pour élaborer un modèle idéal de ce que doit être l'écriture des blogs, la référence aux genres est un moyen d'orienter les pratiques d'écriture, et de donner un degré de réalité à une utopie communicationnelle de transparence et de vérité.

\section{La construction sociale d'un objet de valeurs}

Parler d'une génétique des blogs, c'est mettre à distance l'illusion angélique d'une écriture qui serait spontanément transparente, authentique et naturelle : c'est montrer que tout est contenu, en fait, dans le geste de référence et de convocation de genres littéraires et communicationnels hérités.

Il est important d'insister sur ce point : l'engagement dont il est question est de l'ordre de l'écriture, et non de l'écrit; il est de l'ordre de la disposition de l'utilisateur et non des dispositifs eux-mêmes. Dans le cas des formes éditoriales manipulables sur Internet, il s'établit des manières d'agir, des normes de la pratique, dans une régularité qui est plus de l'ordre d'une normativité morale que d'une catégorisation textuelle. On perçoit clairement ici que le genre ne peut être pensé, dans le cas des blogs, indépendamment du contexte et de la situation sociale. En effet, l'intervention de morales de l'écriture dans les modes d'appropriation et dans les pratiques est liée au développement d'un champ important de représentations axiologiques et idéologiques de ce que le blog est censé permettre et engager. Quand on a affaire à une caractérisation du blog comme genre, on est en présence de la manifestation d'un imaginaire de la forme, d'une représentation idéologique qui a pour prétention d'en fixer l'éthique, et qui se traduit par des pratiques d'écriture mettant en scène cet idéal moral. La référence générique à des modèles textuels - et singulièrement le « journal personnel », qui maximise l'éthique de véracité - engage des pratiques génétiques de la production de textes.

Étienne Candel Université de Paris-Sorbonne-CELSA - GRIPIC 